\title{
Smad7:ß-catenin complex regulates myogenic gene transcription
}

\author{
Soma Tripathi ${ }^{1,2,3}$, Tetsuaki Miyake ${ }^{1,2,3}$ and John C. McDermott (1) $1,2,4$
}

\begin{abstract}
Recent reports indicate that Smad7 promotes skeletal muscle differentiation and growth. We previously documented a non-canonical role of nuclear Smad7 during myogenesis, independent of its role in TGF- $\beta$ signaling. Here further characterization of the myogenic function of Smad7 revealed $\beta$-catenin as a Smad7 interacting protein. Biochemical analysis identified a Smad7 interaction domain (SID) between aa575 and aa683 of $\beta$-catenin. Reporter gene analysis and chromatin immunoprecipitation demonstrated that Smad7 and $\beta$-catenin are cooperatively recruited to the extensively characterized $\mathrm{ckm}$ promoter proximal region to facilitate its muscle restricted transcriptional activation in myogenic cells. Depletion of endogenous Smad7 and $\beta$-catenin in muscle cells reduced ckm promoter activity indicating their role during myogenesis. Deletion of the $\beta$-catenin SID substantially reduced the effect of Smad7 on the $\mathrm{ckm}$ promoter and exogenous expression of SID abolished $\beta$-catenin function, indicating that SID functions as a trans dominant-negative regulator of $\beta$-catenin activity. $\beta$-catenin interaction with the Mediator kinase complex through its Med12 subunit led us to identify MED13 as an additional Smad7-binding partner. Collectively, these studies document a novel function of a Smad7-MED12/13- $\beta$-catenin complex at the $\mathrm{ckm}$ locus, indicating a key role of this complex in the program of myogenic gene expression underlying skeletal muscle development and regeneration.
\end{abstract}

\section{Introduction}

Developmental myogenesis, the process of terminal differentiation of skeletal muscle progenitor cells, consists of a series of well-characterized highly regulated steps that has become a paradigm for lineage acquisition and cellular differentiation ${ }^{1-5}$. During embryogenesis, pluripotent mesodermal stem cells commit to become myogenic progenitor cells ${ }^{6}$. Commitment to the myogenic lineage results in a binary state of either maintenance of proliferative potential and multipotency or, on appropriate cues, withdrawal from the cell cycle, activation of a battery of structural, contractile, and metabolic genes and ultimately formation of multi-nucleated, electrically excitable myofibers ${ }^{1,7,8}$. Also, in adult skeletal muscle,

\footnotetext{
Correspondence: John C. McDermott (jmcderm@yorku.ca)

${ }^{1}$ Department of Biology, York University, Toronto, ON M3J 1P3, Canada

${ }^{2}$ Muscle Health Research Centre (MHRC), York University, Toronto, ON M3J 1P3,

Canada

Full list of author information is available at the end of the article.

Edited by A. Oberst
}

resident stem cells (satellite cells) located between the basal lamina and the plasma membrane of mature muscle fibers ${ }^{9}$ recapitulate this "myogenic program" of differentiation in response to injury or for routine maintenance of the muscle tissue ${ }^{10}$.

How networks of transcriptional regulators exert molecular control over developmental and adult myogenesis has been a prevalent theme in understanding the molecular control of ontogeny, physiology, and pathology of the striated muscle ${ }^{10-12}$. Extensive biochemical and genetic evidence has implicated a family of DNA-binding transcriptional regulatory proteins encoded by the myogenic regulatory factor (MRF) genes, myf5, myod1, myogenin (myog), and mrf4, in myogenesis ${ }^{10,13}$. In conjunction with the proteins encoded by the myocyte enhancer factor 2 (mef2a-d) gene family, the MRF's activate an evolutionarily conserved program of gene expression, which leads to the generation of terminally differentiated skeletal muscle cells ${ }^{14-16}$. Understanding the trans-acting factors contributing to this process has been aided in tandem by

\section{(c) The Author(s) 2019}

(c) (i) Open Access This article is licensed under a Creative Commons Attribution 4.0 International License, which permits use, sharing, adaptation, distribution and reproduction cc) in any medium or format, as long as you give appropriate credit to the original author(s) and the source, provide a link to the Creative Commons license, and indicate if changes were made. The images or other third party material in this article are included in the article's Creative Commons license, unless indicated otherwise in a credit line to the material. If material is not included in the article's Creative Commons license and your intended use is not permitted by statutory regulation or exceeds the permitted use, you will need to obtain permission directly from the copyright holder. To view a copy of this license, visit http://creativecommons.org/licenses/by/4.0/. 
extensive analysis of the cis-regulatory elements of muscle-restricted, differentiation-induced genes such as the muscle creatine kinase $(\mathrm{ckm})$ gene $^{17-19}$.

In addition to the central role played by the MRF/MEF2 axis in myogenesis ${ }^{20}$, other transcription factors have been implicated in the control of myogenic differentiation such as Six 1 and $4^{21-23}, \mathrm{AP}-1^{24,25}, \beta$-catenin ${ }^{26-28}$, and $\operatorname{Smad} 7^{29-31}$. Since our initial observations of the promyogenic role of Smad7 in cultured muscle cells ${ }^{29}$, we have documented a novel nuclear role for Smad7 in muscle that is essentially independent of its "canonical" role as a repressor of transforming growth factor (TGF) $\beta$ signaling ${ }^{31}$. In addition, other groups have documented an in vivo role for Smad7 in the skeletal muscle ${ }^{30}$. Understanding the nature of the ancillary role played by Smad7 and other transcriptional regulators at muscle-restricted genes is therefore of some importance for our overall understanding of the molecular programming of myogenic identity.

In view of the "non-canonical" nuclear role of Smad7 alluded to above, the mechanistic basis by which it contributes to the myogenic differentiation program is so far incompletely understood. We were intrigued by a report identifying a protein-protein interaction (PPI) between Smad7 and $\beta$-catenin in human prostate cancer cells ${ }^{32}$ since both are known, independently, to be key regulators of muscle gene expression in a variety of contexts. We therefore assessed this putative PPI in cultured muscle cells. Our data support a robust interaction between Smad7 and $\beta$-catenin that contributes to the transcriptional control of a key myogenic promoter $(\mathrm{ckm})$. We further extended these observations in characterizing the recruitment of Mediator components (Med12/13) by the $\beta$-catenin/Smad7 complex, thus connecting the muscle transcriptosome assembled on enhancers such as $c k m$ to the basal transcription machinery. Integration of the Smad7- $\beta$-catenin complex into the network of proteins regulating the "myogenic program" expands our understanding of the unique molecular wiring encoding myogenic differentiation, growth and repair.

\section{Materials and methods \\ Cell culture}

C2C12 myoblasts were obtained from the American Type Culture Collection. Cells were cultured in growth medium (GM) consisting of high-glucose Dulbecco's modified Eagle's medium (DMEM, Gibco), 10\% fetal bovine serum (FBS), and L-Glutamine (HyClone) supplemented with $1 \%$ penicillin-streptomycin (Invitrogen, ThermoFisher). Myotube formation was induced by replacing GM with differentiation medium (DM), consisting of DMEM supplemented with $2 \%$ horse serum (Atlanta Biologicals) and $1 \%$ penicillin-streptomycin.
Cells were maintained in an incubator at $95 \%$ humidity, $5 \% \mathrm{CO}_{2}$, and $37^{\circ} \mathrm{C}$.

\section{Transfections}

For ectopic protein expression, cells were transfected using the calcium phosphate precipitation method for transcription reporter assays. Cells were re-fed $16 \mathrm{~h}$ posttransfection and harvested. For small interfering RNA (siRNA) experiments, cells were transfected with Lipofectamine 2000 (Life Technologies) using instructions provided by the manufacturer and harvested $48 \mathrm{~h}$ later, unless otherwise indicated.

\section{Gene silencing}

MISSION siRNA (Sigma-Aldrich) for rat and mouse ctnnb1 siß-catenin\#1 (SASI_Rn01_00099925), si $\beta$-catenin \#2 (SASI_Rn01_00099923), siß-catenin\#3 (SASI_Rn01_00 099924), siSmad7\#1 (SASI_Mm02_00290887), siSmad7\#2 (SASI_Mm02_00290886), siSmad7\#3 (SASI_Mm02_0029 0885) and universal scrambled siRNA (SIC001) were used at $75 \mathrm{nM}$ concentrations.

\section{Plasmids}

Expression plasmids for Myc-His-tagged full-length Smad7, $\beta$-catenin-myc, transcription reporter assay constructs $p c k m$-luc have been described previously ${ }^{29,31,33}$. $\beta$-catenin mutant expression plasmids were constructed by the ligation of PCR-amplified nucleotides corresponding to the indicated amino acid (aa) regions (aa575-683, aa1-574) at Hind III and Xho I sites of pcDNA3-EYFP or pcDNA3-3Xflag-8XHis, respectively. Constructs for expression of glutathione-S-transferase (GST)-fused $\beta$-catenin fragments were described previously $^{34}$.

\section{Transcription reporter gene assays}

Transcriptional reporter assays were performed using luciferase reporter plasmids along with expression constructs (indicated in the figure legends) and a Renilla plasmid (pRL-Renilla, Promega) as an internal control. Cells were washed with $1 \times$ phosphate-buffered saline and harvested in Luciferase Lysis Buffer (20 mM Tris pH 7.4 and $0.1 \%$ Triton X-100). Enzymatic activity was measured in each sample on a luminometer (Lumat LB, Berthold) using Luciferase assay substrate (E1501; Promega) or Renilla assay substrate (E2820; Promega). Luciferase activity values obtained were normalized to Renilla activity in the same cell extracts and expressed as fold activation to the control.

\section{Nuclear and cytoplasmic extraction}

Nuclear and cytoplasmic extraction was obtained using the NE-PER Kit (78833; Thermo Scientific), as per the 
instructions provided by the manufacturer. Immunoblotting of extracellular signal-regulated kinase and c-Jun was used as the positive control for cytoplasmic and nuclear fractions, respectively.

\section{Western blot analysis}

Total cellular protein extracts were prepared in NP-40 lysis buffer $(0.5 \% \quad(\mathrm{vol} / \mathrm{vol})), \quad 50 \mathrm{mM}$ Tris- $\mathrm{HCl} \quad(\mathrm{pH}$ 8), $150 \mathrm{mM} \mathrm{NaCl}, 10 \mathrm{mM}$ sodium pyrophosphate, $1 \mathrm{mM}$ EDTA ( $\mathrm{pH} 8$ ), and $0.1 \mathrm{M} \mathrm{NaF}$ supplemented with $1 \times$ protease inhibitor cocktail (P-8340; Sigma) and $0.5 \mathrm{mM}$ sodium orthovanadate. Protein concentrations were determined by a standard Bradford assay. Equivalent amounts of protein were denatured in sodium dodecyl sulfate (SDS) loading buffer at $100^{\circ} \mathrm{C}$ for $5 \mathrm{~min}$ and then run in SDS-polyacrylamide gels, followed by electrophoretic transfer to an Immobilon-FL polyvinylidene difluoride membrane (Millipore) as directed by the manufacturer. Blots were incubated with blocking buffer that consisted of $5 \%$ milk in Tris-buffered saline (TBS)-T (10 mM Tris- $\mathrm{HCl}, \mathrm{pH} 8.0,150 \mathrm{mM} \mathrm{NaCl}, 0.1 \%$ Tween 20$)$ prior to the incubation with primary antibody at $4{ }^{\circ} \mathrm{C}$ overnight with gentle agitation. After three washes with TBS-T, appropriate horseradish peroxidase-conjugated secondary antibody (BioRad, 1:2000) were added for $2 \mathrm{~h}$ at room temperature. Protein/antibody immune-complexes were detected with Enhanced Chemiluminescence western blotting substrate (Pierce, ThermoFisher).

\section{Antibodies}

Rabbit monoclonal for $\alpha \operatorname{Smad} 7$ (ab124890) and polyclonal for $\alpha$ MED13 (ab76923) and $\alpha$ MED12 (ab70842) were purchased from Abcam. A rabbit polyclonal antibody was raised against GST-Smad7 according to the protocol approved by York University of Animal Care Committee. This was used for endogenous Smad7 immunoprecipitation (IP) and detection in cellular and nuclear extract. $\alpha \beta$-Catenin (pAb9562) and ChIP-grade $\alpha$ Flag antibody (mAb 14793S) were purchased from Cell signaling. Monoclonal $\alpha$ Flag antibody (F1804) was from Sigma. $\alpha$ Myc (9E10), $\alpha \mathrm{MyHC}$ (MF20), and $\alpha$ Myogenin (F5D) were purchased from DSHB. $\alpha$ Actin (Sc1616), $\alpha \mathrm{MyoD}$ (sc304), and $\alpha C K M$ (sc-69878) were purchased from Santa Cruz.

\section{Co-immunoprecipitation (Co-IP)}

For Co-IP, cells were harvested, and proteins were extracted as described above. IP was performed using an ImmunoCruz Optima Kit (Santa Cruz Biotechnology) according to the manufacturer's instructions. Eluates were analyzed by western blotting as described above.

\section{Live-cell imaging}

C2C12 cells were seeded onto the glass-bottom dishes (MatTek Corp). The cells were transfected for the expression of fluorescent-tagged proteins. Before imaging the cells, the media was replaced for FluoroBrite DMEM (Thermo Fisher Scientific) supplemented with 10\% FBS. To mark nuclei, Hoechst 33342 (Sigma-Aldrich) was added to $2.5 \mu \mathrm{M}$ into the media. After $30 \mathrm{~min}$, the stained cells were visualized by a Carl Zeiss Spinning disc system (Zeiss Observer Z1 with Yokogawa CSU-X1 and AxioCam MRm camera) in the environment chamber $\left(37^{\circ} \mathrm{C}\right.$, $5 \% \mathrm{CO}_{2}$ ). The raw images were processed by ZEN (Carl Zeiss) to obtain pseudo-colored micrographs.

\section{Chromatin immunoprecipitation (ChIP)}

C2C12 myoblasts or Smad7-Flag ectopically expressing myoblasts were crosslinked with $1 \%$ formaldehyde followed by sonication to shear DNA strands attached to the protein. Smad7/ $\beta$-catenin was immunoprecipitated with Flag or $\beta$-catenin antibody to obtain the protein-DNA complex. IP with IgG antibody served as controls. Immunoprecipitated DNA was reverse crosslinked, purified, and subjected to quantitative PCR (qPCR) using primers specific for the $c k m$ promoter. Primers specific to gapdh was used as controls for $c k m$ enrichment.

\section{GST pull down assay}

For GST pull down assays, GST-Smad7 and 6XHis$\beta$-catenin fusion proteins were produced in bacteria using standard protocol. Briefly, GST-Smad7- and 6XHis$\beta$-catenin-expressing cells were sonicated and the proteins were purified with glutathione-agarose beads (Sigma-Aldrich G-4510). Protein concentrations were estimated by SDS-polyacrylamide gel electrophoresis and following Coomassie blue staining, using BSA for comparative estimation. GST- $\beta$-catenin fragments corresponding to aa (full length (FL), 1-100, 120-683, $422-683,575-696,575-683,1-574)$ were utilized for mapping study. Five micrograms of GST FL Smad7, $\beta$-catenin (or the molar equivalent of the smaller GST, GST- $\beta$-catenin fragments), and $25 \mu \mathrm{l}$ glutathione-agarose beads (50\% slurry) were incubated in $600 \mu$ NETN buffer (100 mM NaCl, $20 \mathrm{mM}$ Tris- $\mathrm{HCl}(\mathrm{pH} 8) 0.5 \mathrm{mM}$ EDTA, $0.5 \%$ (vol/vol) NP-40) overnight at $4{ }^{\circ} \mathrm{C}$. In experiments where GST- $\beta$-catenin was used to co-precipitate Smad7, GST-Smad7 was thrombin (GE Healthcare-27-0846-01) digested to yield the immune-complex without the GST tag $(10 \mu \mathrm{l}$ thrombin per $\mathrm{mg}$ fusion protein was incubated at room temperature for $17 \mathrm{~h}$ ), and eluates were analyzed by western blotting.

\section{Results}

Smad7 and $\beta$-catenin are co-expressed and interact in myogenic cells

A time course analysis of $\beta$-catenin and myogenic markers during $\mathrm{C} 2 \mathrm{C} 12$ differentiation indicated that $\beta$-catenin expression is enhanced along with myosin 
heavy chain $(\mathrm{MyHC})$, muscle creatine kinase (CKM), and myogenin levels during differentiation (Fig. 1a). Endogenous Smad7 protein levels increased during differentiation as compared to myoblasts in growth conditions (Fig. 1b). Immunofluorescence analysis of Smad7- and $\beta$-catenin-expressing $\mathrm{C} 2 \mathrm{C} 12$ cells indicates that both Smad7 and $\beta$-catenin are localized in the nucleus; interestingly, two different patterns of localization were observed. $\beta$-Catenin exhibited a diffuse pattern of localization within the nucleus in some cells while in others a very defined co-localization occurred in nuclear puncta (Fig. 1c). A cytoplasmic and nuclear fractionation demonstrated that Smad7 and $\beta$-catenin are abundant in both fractions (Fig. 1d). Based on a previous report indicating a PPI between $\beta$-catenin and Smad7 in human prostate cancer cells $(\mathrm{PC}-3 \mathrm{U})^{32}$, we assessed whether Smad7 and $\beta$-catenin form a complex under these cellular conditions in cultured muscle cells and muscle tissue (mouse tibialis anterior (TA)). Total protein lysates from mouse TA and $\mathrm{C} 2 \mathrm{C} 12$ muscle cells were subjected to CoIP with $\beta$-catenin and Smad7 antibodies. Interestingly, Smad7 and $\beta$-catenin were precipitated together in muscle tissue (TA) and in muscle cells (Fig. 1e). The interaction was further confirmed by ectopically expressing Smad7-Flag followed by detection of $\beta$-catenin in the Co-IP (Fig. 1f).

\section{Smad7 physically interacts with $\beta$-catenin}

As the Co-IP analysis indicated Smad7 and $\beta$-catenin were in the same complex, we next clarified whether this was a direct interaction by utilizing GST pull down assays with bacterially expressed purified proteins. GST-Smad7 and $6 x$ Histidine (6xHis)- $\beta$-catenin fusion protein were produced to conduct the assay. A Coomassie stained blot confirmed the purification of the fusion proteins (Fig. 2a). $\beta$-catenin immunoprecipitated with GST-Smad7 but not with GST-conjugated beads indicated that this interaction was direct (Fig. 2b). Next, we determined the Smad7 interaction domain (SID) on $\beta$-catenin. The 781 aa sequence of $\beta$-catenin gives rise to a structure consisting of several characteristic repeats, termed armadillo repeats, each approximately 40 aa's long. N-terminal (NTD) and C-terminal (CTD) domains flank either end of the armadillo repeats. Helix-C indicates a conserved helix

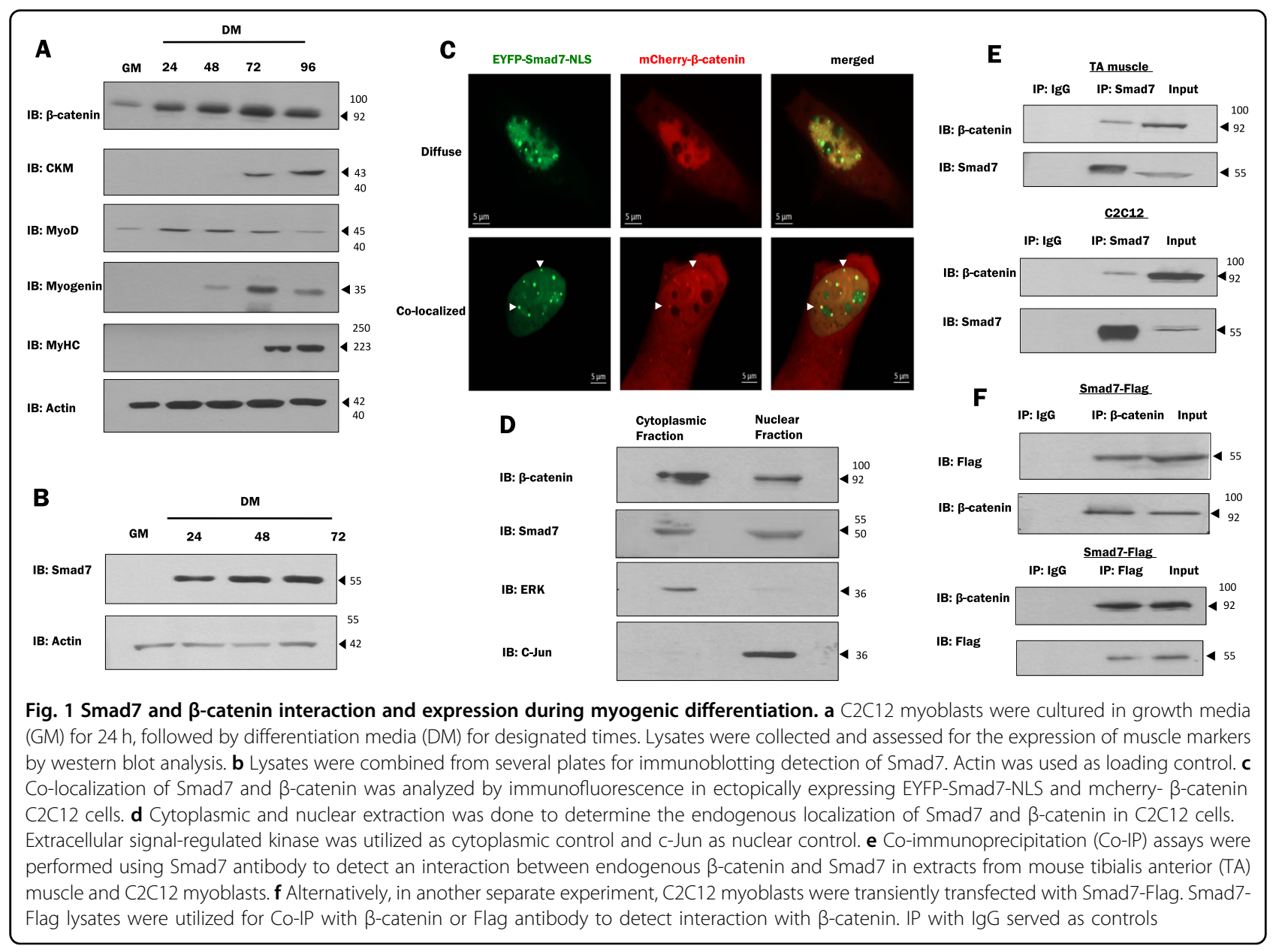




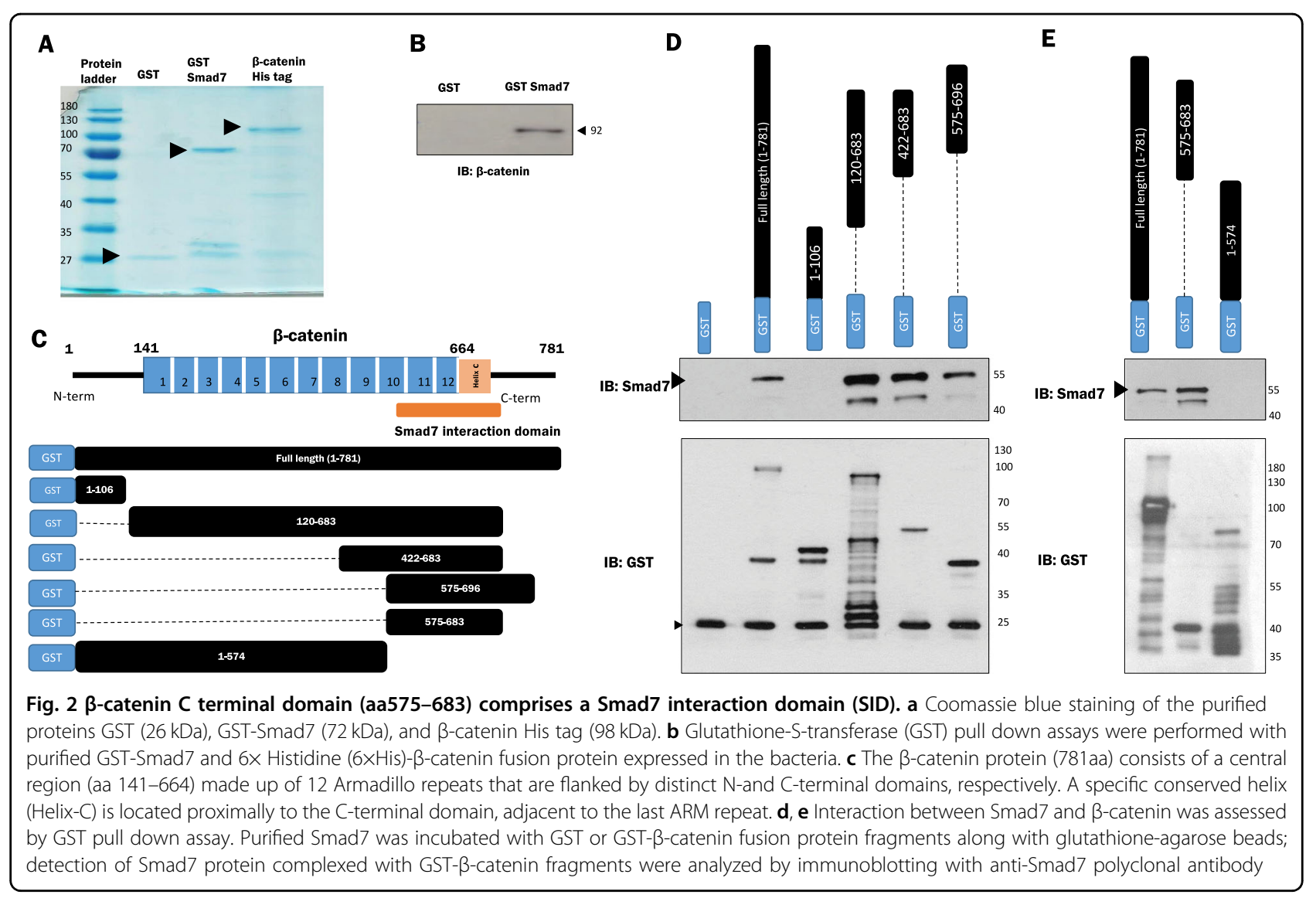

located adjacent to the last ARM repeat proximal to the CTD $^{35}$ (Fig 2c). GST- $\beta$-catenin protein fragments corresponding to aa's $1-106,120-683,422-683,575-696$, and the FL were utilized for determining the interacting region with Smad7. Smad7 interacted with FL $\beta$-catenin (as we previously identified) and $\beta$-catenin aas 120-683, 422-683 and 575-696. Smad7 did not interact with $\beta$-catenin aa1-106 therefore indicating that the region between aa575 and aa683 (Fig. 2d) is required for this interaction. To further refine this, we used additional GST- $\beta$-catenin fragments, GST- $\beta$-catenin aa575-683 and GST- $\beta$-catenin aa1-574 and conducted the interaction assay. In agreement with the above results, we observed that Smad7 interacted with $\beta$-catenin aa575-683 and not with aa1-574 leading us to conclude that the SID on $\beta$-catenin lies between aa575 and aa683 (Fig. 2e). This region spans the 10th armadillo repeat of $\beta$-catenin and a partial region extending into the CTD. Previously, this CTD domain has been associated with $\beta$-catenin transactivation properties and has been shown to interact with MED12, TBP (TATA-binding protein), CBP (CREBbinding protein/p300), and proteins associated with chromatin regulation ${ }^{35}$.
Smad7 and $\beta$-catenin are enriched on the muscle-specific ckm promoter proximal region

The regulatory region of the $c k m$ gene $(c k m)$ has been extensively characterized and has served as a paradigm for tissue-restricted transcriptional control during myogenesis $^{17,36}$. Our laboratory previously documented that Smad7 associates with the promoter proximal elements of $c \mathrm{~km}^{29}$. We therefore used this as a test promoter to assess the function of the $\beta$-catenin:Smad7 interaction and to interrogate the role of this complex during myogenic differentiation. First, ChIP coupled with qPCR for $\mathrm{ckm}$ and gapdh (control) was utilized to assess whether Smad7 and $\beta$-catenin are enriched on the $c k m$ promoter proximal region. In the absence of a Smad7 antibody efficacious for ChIP, Smad7 enrichment was confirmed by Flag-Smad7 recruitment to the $\mathrm{ckm}$ promoter (Fig. 3a). Additionally, endogenous $\beta$-catenin is enriched at the $\mathrm{ckm}$ promoter in myogenic cells (Fig. 3b). Next, we utilized reporter gene assays in which Smad7 and $\beta$-catenin were exogenously expressed in $\mathrm{C} 2 \mathrm{C} 12$ cells along with a $c k m$ ( -1082 to -1262) promoter fragment driving a firefly luciferase reporter gene. These data indicate that Smad7 and $\beta$-catenin transactivate this promoter region both alone 


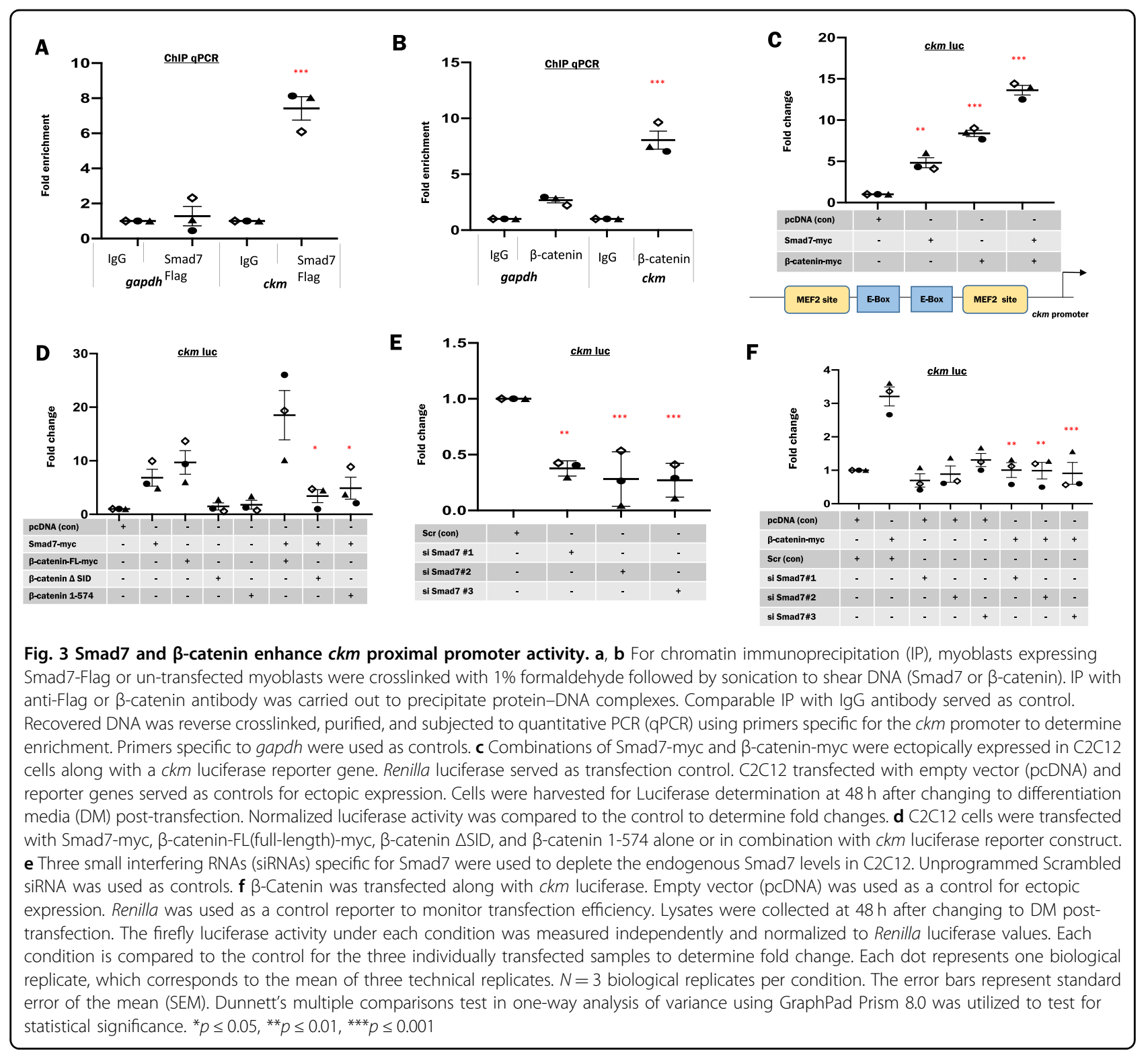

and in combination (Fig. 3c). Based on the interaction mapping, a mammalian expression vector for $\beta$-catenin without the SID (deletion of aa575-683) was constructed. Initially, we performed reporter gene assays of the activity of these $\beta$-catenin constructs using the TOP flash luciferase reporter gene, which acts both as a $\beta$-catenin reporter and a general Wnt signaling pathway reporter as it contains 7 TCF/LEF consensus sites in which TCF/LEF proteins bind to but cannot activate the reporter gene without $\beta$-catenin. Our data indicated that the FL $\beta$-catenin activated TOP flash, whereas $\beta$-catenin $1-574$ and $\beta$-catenin $\triangle$ SID could minimally activate TOP flash compared to the FL $\beta$-catenin (supplementary Fig. S1). Further, when $\beta$-catenin was ectopically expressed with Smad7, there was an enhancement in TOP flash promoter activity, whereas there was no change in TOP flash activity in conditions where $\operatorname{Smad} 7$ was expressed in combination with $\beta$-catenin $1-574$ and $\beta$-catenin $\triangle$ SID. These data revealed that, while Smad7 was able to enhance the function of the intact $\beta$-catenin, it did not cooperate with $\beta$-catenin $1-574$ and $\beta$-catenin $\triangle$ SID in reporter gene assays (supplementary Fig. S1). We next deleted the SID domain to test whether it might prevent the cooperative transactivation mediated by Smad7 and $\beta$-catenin. To test this idea, Smad7, FL $\beta$-catenin ( $\beta$-catenin-FL), $\beta$-catenin (aa1-574, $\Delta$ SID) along with a $\mathrm{ckm}$ luciferase construct were ectopically expressed in $\mathrm{C} 2 \mathrm{C} 12$ cells alone or in combination. These data indicate that, while Smad7 and the $\beta$-catenin-FL enhanced the $c k m$ promoter, this activity was substantially reduced in cells 
expressing Smad7 in combination with $\beta$-catenin aa1-574 or $\beta$-catenin $\Delta$ SID (Fig. 3d). These data support the conclusion that the $\beta$-catenin SID is crucial for the cooperative interaction of Smad7 and $\beta$-catenin on the $c k m$ promoter. To address this cooperativity in a different way, we next investigated the effect of perturbation in Smad7 expression on the function of $\beta$-catenin on the ckm promoter using siRNA technology. Endogenous Smad7 levels were depleted using three independent siRNAs (supplementary Fig. S2) and reporter gene analysis indicated a pronounced decrease in $\mathrm{ckm}$ promoter activity when compared to the control (Fig. 3e). Ectopic expression of $\beta$-catenin in Smad7-depleted cells exhibited a reduction in $\mathrm{ckm}$ promoter induction consistent with the interpretation that $\beta$-catenin requires Smad7 in order to affect promoter activation (Fig. 3f). To further test this idea, we correspondingly depleted $\beta$-catenin expression using three independent siRNAs. Western blot analysis verified a considerable reduction in protein levels of $\beta$-catenin using two different siRNAs (si\#2 and \#3) as compared to controls. Under conditions in which endogenous $\beta$-catenin was depleted, we observed that Smad7 mediated $\mathrm{ckm}$ promoter activation was markedly reduced (Fig. 4a). These data indicate that both Smad7 and $\beta$-catenin cooperatively activate the $\mathrm{ckm}$ promoter. Previously, it was shown that $\mathrm{MyoD}$, the archetypal $\mathrm{MRF}^{37}$, interacts with $\beta$-catenin ${ }^{38}$. To test this hypothesis, endogenous $\beta$-catenin levels were depleted in $\mathrm{C} 2 \mathrm{C} 12$ using two different siRNAs. MyoD and Smad7-myc either alone or in combination were then transfected along with the $\mathrm{ckm}$ reporter gene in $\beta$-catenin-depleted cells. This analysis revealed that $c k m$ promoter activity increased by Smad7 or/and MyoD was reduced by depletion of $\beta$-catenin (Fig. $4 \mathrm{~b})$. These results further support the conclusion that $\beta$-catenin enhances Smad7- and MyoD-driven potentiation of $\mathrm{ckm}$ promoter activity. These observations in combination with previously reported data indicate that the Smad7: $\beta$-catenin interaction may be tethered by MyoD and is required for full $c k m$ promoter activation.

\section{The minimal SID on $\beta$-catenin functions as a transdominant inhibitor of $\beta$-catenin activity}

Since our data suggested that the SID is crucial for the function of Smad7 and $\beta$-catenin on the $c k m$ promoter, we next considered the possibility that this domain of $\beta$-catenin might function as a more general transdominant repressor (dominant negative) of endogenous $\beta$-catenin activity. To test this idea, we constructed a mammalian expression plasmid for tagged $\beta$-catenin aa575-683. Smad7 and $\beta$-catenin were ectopically expressed in $\mathrm{C} 2 \mathrm{C} 12$ cells along with increasing concentrations of either EYFP- $\beta$-catenin aa575-683 (EYFPSID) or Flag $\beta$-catenin aa575-683 (Flag-SID). Reporter gene analysis revealed that $\mathrm{ckm}$ promoter activity was significantly reduced in Smad7- and $\beta$-catenin-expressing cells in the presence of either EYFP-SID or Flag-SID (Fig $5 a)$. These data indicate that the $\beta$-catenin SID can function as a dominant-negative inhibitor of $\beta$-catenin function. A similar analysis using a sprrla promoter luciferase reporter gene that is not regulated by $\mathrm{MyoD}$ (data not shown) was unaffected by SID expression, suggesting that the effect of SID on $\mathrm{ckm}$ is specific (supplementary Fig. S3). We subsequently assessed the effect of the SID domain on myogenesis by analyzing the protein levels of myogenic markers with and without SID expression. Immunoblot analysis from ectopically expressed SID in C2C12 cells exhibited reduced protein levels of CKM and Myogenin as compared to controls indicating an overall repression of myogenesis by exogenous SID expression (Fig. 5b). Collectively these data suggested that the $\beta$-catenin SID can inhibit the activity of endogenous $\beta$-catenin and the myogenic differentiation program.

\section{Smad7: $\beta$-catenin complex interact with the Mediator kinase complex subunits (MED13 and 12)}

Previous studies ${ }^{39}$ and our unpublished observations confirm that $\beta$-catenin directly interacts with MED12. Since it is established that MED12 and MED13 form an integral part of the mediator kinase module ${ }^{39,40}$, we hypothesized that the composite function of the Smad7: $\beta$-catenin interaction might be to recruit the MED kinase module. This idea is supported by our Co-IP analysis in which we observed that Smad7 and MED13 are in the same precipitated protein complex (Fig. 6a). Furthermore, we also validated the previously reported MED12: $\beta$-catenin interaction (Fig. 6b). Finally, we observed that the Smad7:MED13 interaction was disrupted when endogenous $\beta$-catenin was depleted (Fig. 6c, d). The Smad7:MED13 interaction thus constitutes a novel observation in characterizing Smad7 as a component of the transcriptional machinery-linking promoter activity to the Mediator kinase complex.

\section{Discussion}

\section{Smad7: $\beta$-catenin as an essential component of muscle enhanceosomes}

Despite a considerable body of literature implicating Wnt- $\beta$-catenin signaling in muscle differentiation ${ }^{26,41-43}$, there is a surprising lack of mechanistic insight into how it fulfills this role. Moreover, recent in vitro and in vivo data implicating Smad7 in the control of muscle gene expression is also not understood mechanistically. Interestingly, in one study ${ }^{44}$ a correlation between TGF- $\beta$ and Wnt signaling was investigated where TGF- $\beta 1$ was observed to control the differentiation of fibroblasts to myofibroblasts by upregulating Wnt signaling. Here we provide evidence of a direct functional interaction 


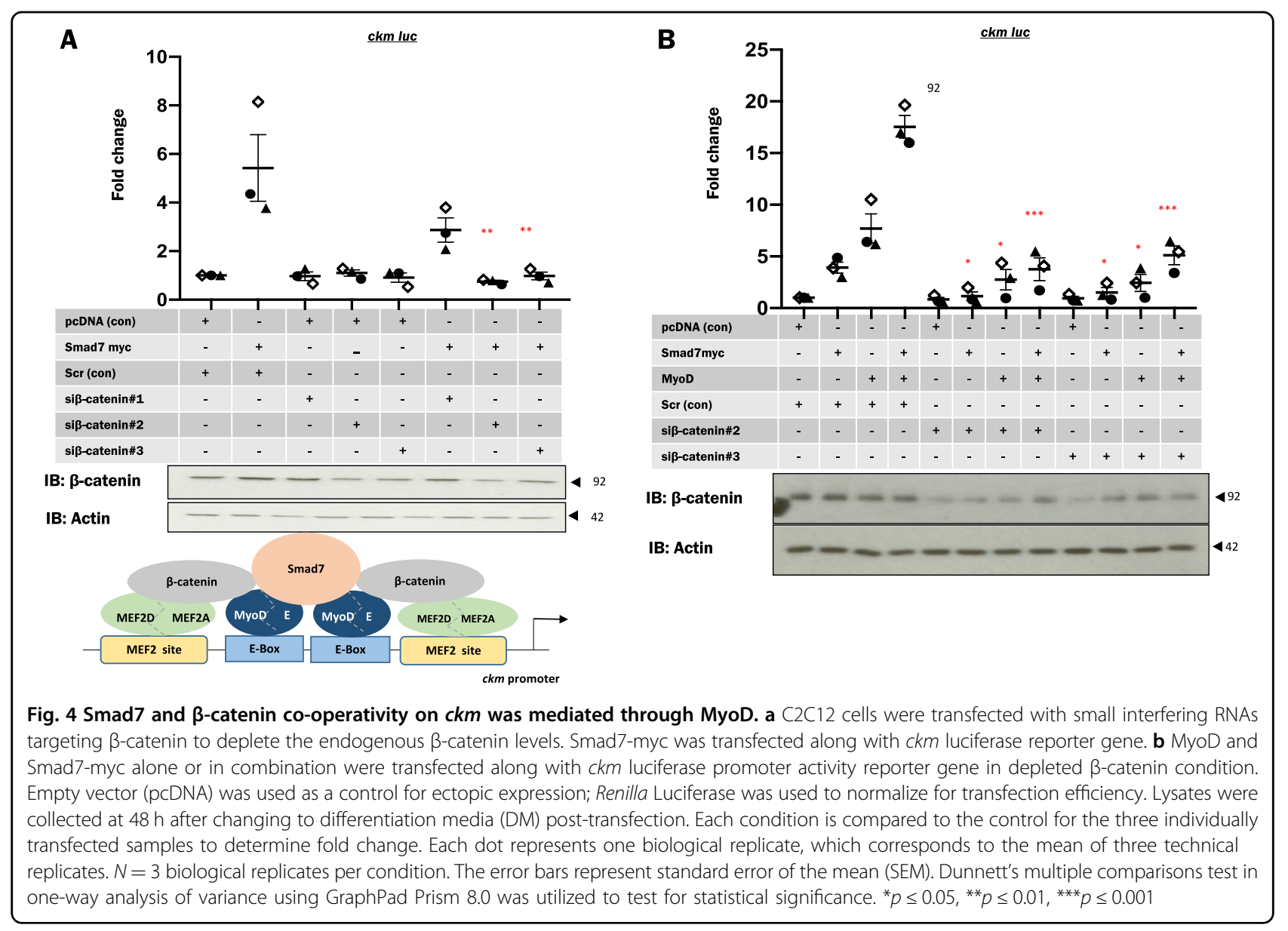

between Smad7 and $\beta$-catenin that serves a fundamental role in recruiting Mediator components to the wellcharacterized $\mathrm{ckm}$ gene. Our data indicate that $\beta$-catenin is inefficiently recruited to the $c k m$ gene enhancer in muscle cells when Smad7 is depleted and corresponding loss of $\beta$-catenin recruitment renders this gene incapable of responding to essential differentiation cues (Fig. 7). Here we present evidence indicating that a Smad7: $\beta$-catenin complex is a critical part of the transcriptional machinery at a key muscle promoter proximal element, being intrinsically necessary as a co-regulator for the MRFs in the myogenic gene expression program.

\section{Mediator recruitment to $\mathrm{ckm}$ by $\beta$-catenin/Smad7}

The Mediator complex was initially characterized in budding yeast ${ }^{45}$ and has since been established as fulfilling an essential function in RNA polymerase II-mediated gene transcription from flies to mammals ${ }^{46}$. The multisubunit compositional complexity of the Mediator holocomplex has proved a substantial challenge to define the full extent of its properties but what is apparent is that its fundamental role is to provide a functional bridge between transcriptional regulatory proteins bound to gene enhancers and the general transcription machinery assembled at core promoters ${ }^{47-53}$. The interaction of different Mediator subunits with a variety of transcription factors thus allows a myriad of cellular signaling events that converge on the transcription factors to be subsequently relayed to the transcriptional machinery and ultimately programs of gene transcription ${ }^{54}$. Thus, this essential activity in promoting signal-dependent transcriptional pre-initiation complex (PIC) assembly and stabilization renders many Mediator subunits essential for life, since gene targeting in mice has revealed that many subunits prove embryonic lethal when deleted and in yeast all Pol II-regulated genes are dependent on Mediator $^{52,55-57}$. The experimental dissection of Mediator has been aided by the characterization of four relatively stable sub-complexes that have been designated as the head, middle, tail, and kinase modules. In our study, we have

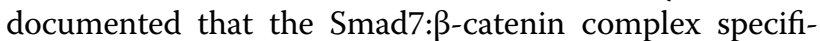
cally associates with the MED12 and 13 subunits of the Mediator kinase module. The kinase domain is speculated to fulfill a transient regulatory function in Mediator by promoting recruitment of all four Mediator domains to enhancers which then transitions to a core promoter- 


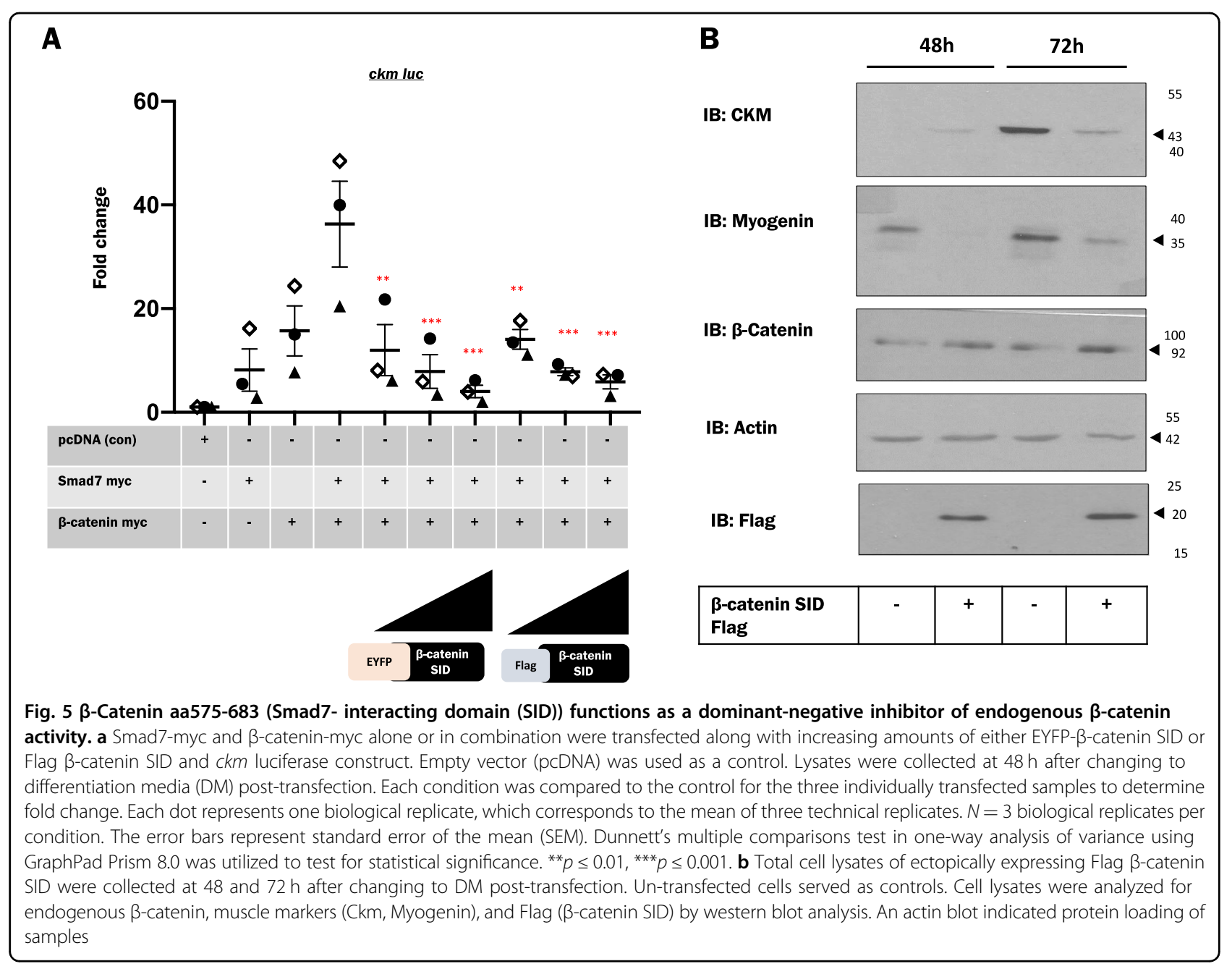

bound Mediator complex in which the kinase module is absent. We therefore propose that Mediator recruitment to the $c k m$ enhancer is mediated by the Smad7: $\beta$-catenin complex in muscle cells (Fig. 7).

\section{Implications of $\beta$-catenin:Smad7 interaction in Rhabdomyosarcoma (RMS)}

In view of the role played by $\beta$-catenin: $\operatorname{Smad} 7$, it is perhaps worth consideration of the implications of this in the context of the pathology of RMS, a soft tissue pediatric cancer with features of muscle ${ }^{58}$. Previously, we reported that constitutive glycogen synthase kinase 3 (GSK3) activity is a feature of the embryonal form of RMS (there are two general categories: alveolar (ARMS) and embryonal (ERMS)). The result of this GSK3 activity is perpetual proteosomal degradation of $\beta$-catenin since this is the primary function of the APC-GSK3 complex in canonical Wnt signaling ${ }^{59}$. Upon Wnt signaling stimulation, GSK3 activity is substantially reduced and proteosomal degradation of $\beta$-catenin ceases, resulting in its cytoplasmic accumulation and translocation to the nucleus where it activates transcription in combination with other transcriptional regulators, such as TCF/LEF. Based on these previous observations and the current study, it is worth speculating that the absence of nuclear $\beta$-catenin function due to constitutive GSK3 activity contributes to the differentiation defect in ERMS. Promoting terminal differentiation of the myo-like cells in RMS is seen as a potentially effective therapeutic target, since differentiation, by nature, results in cell cycle withdrawal and cessation of proliferation which would cause tumor regression. There are several lines of indirect evidence supporting this idea; we recently reported that Myogenin is expressed at high levels in RMS but, despite the role of this MRF as a key terminal effector of the myogenic differentiation program, it is functionally inactive in these cells. It is therefore possible that the lack of nuclear $\beta$-catenin underlies this functional impairment due to the failure to recruit Mediator to connect Myogenin function to PIC assembly in these ERMS cells. In support of this possibility, we observed that Smad7: $\beta$-catenin is tethered to muscle promoters by its 

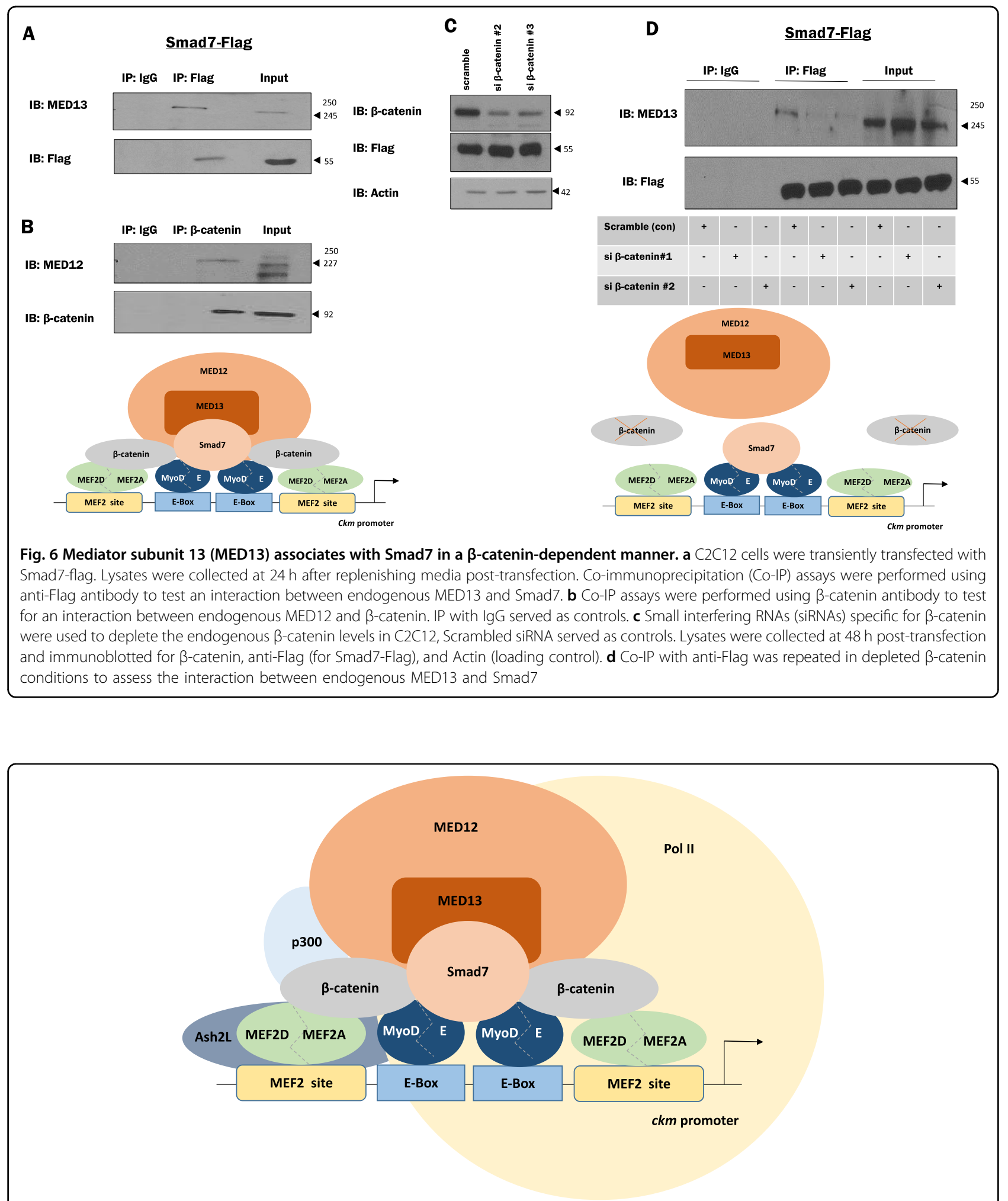

Fig. 7 Proposed model of Smad7: $\beta$-catenin integration into the transcriptional holocomplex on the ckm proximal promoter region. The core $\mathrm{ckm}$ promoter proximal region contains essential binding sites for MEF2 proteins and MyoD/E protein complexes. $\beta$-catenin has also been previously implicated in binding to MyoD and MEF2 transcription factors. The trithorax group protein Ash2L was previously established as one of the interacting partners of MEF2D/2A. Based on the published work and the Smad7:ß-catenin:MED12/13 contribution identified herein, an integrated model of the transcriptional regulation of the $\mathrm{ckm}$ gene is proposed 
interaction with MRFs (such as MyoD and Myogenin) bound to $\mathrm{E}$ boxes on muscle promoter/enhancer regions. Another line of circumstantial evidence supporting this notion is that pharmacological GSK3 inhibitors have been reported to force RMS cells into a more differentiated cellular phenotype, suggesting that re-constitution of $\beta$-catenin function in RMS cells promotes their differentiation and could potentially be antitumorigenic. Further clarification of the role of $\beta$-catenin:Smad7 as a therapeutic target in RMS is therefore warranted.

\section{Further implications of $\mathrm{p} 38$ mitogen-activated protein kinase (MAPK) signaling on $\mathrm{ckm}$ regulation}

Phospho-dependent protein interactions are a signature of $\beta$-catenin function. There is ample evidence of kinasemediated phosphorylation modulating the affinity of PPIs in the canonical Wnt pathway, resulting in important outcomes for Wnt-dependent target gene activation ${ }^{33}$. Specifically, we recently reported that a p38 MAPKdependent interaction with the MEF2 transcription factor enhances $\beta$-catenin nuclear retention and activity ${ }^{33}$. During differentiation, MyoD and MEF2 bind musclespecific promoters and enhancers, leading to the recruitment of co-activators (including p300) and the basal transcriptional machinery to establish a transcriptionally poised promoter. The previously implicated mechanism is that p38 MAPK activates $c k m$ expression by phosphorylation-dependent recruitment of the histone methyltransferase Ash2L by MEF2D ${ }^{60}$. In view of the current observations, it is also possible that p38 MAPK may promote $\beta$-catenin recruitment to $\mathrm{ckm}$ through its interaction with MEF2.

In summary, we have documented a PPI between Smad7 and $\beta$-catenin that may serve a fundamental role in the control of myogenic gene transcription. These observations may have implications for our understanding of the molecular control of myogenic differentiation during embryonic development and adult muscle regeneration.

\section{Acknowledgements}

This work was supported by the Canadian Institutes of Health Research (102688 to J.C.M.). J.C.M. is supported by the McLaughlin Research Chair, York University.

\section{Author details \\ 'Department of Biology, York University, Toronto, ON M3J 1P3, Canada. ${ }^{2}$ Muscle Health Research Centre (MHRC), York University, Toronto, ON M3J 1P3, Canada. ${ }^{3}$ Centre for Research in Biomolecular Interactions (CRBI), York University, Toronto, ON M3J 1P3, Canada. ${ }^{4}$ Centre for Research in Mass Spectrometry (CRMS), York University, Toronto, ON M3J 1P3, Canada}

\section{Conflict of interest}

The authors declare that they have no conflict of interest.

\section{Publisher's note}

Springer Nature remains neutral with regard to jurisdictional claims in published maps and institutional affiliations.
Supplementary Information accompanies this paper at (https://doi.org/ 10.1038/s41419-019-1615-0).

Received: 3 December 2018 Revised: 30 March 2019 Accepted: 23 April 2019

Published online: 16 May 2019

\section{References}

1. Mok, G. F. \& Sweetman, D. Many routes to the same destination: lessons from skeletal muscle development. Reproduction 141, 301-312 (2011).

2. Musumeci, G. et al. Somitogenesis: from somite to skeletal muscle. Acta Histochem. 117, 313-328 (2015).

3. Chal, J. \& Pourquie, O. Making muscle: skeletal myogenesis in vivo and in vitro. Development 144, 2104-2122 (2017).

4. Toto, P. C., Puri, P. L. \& Albini, S. SWI/SNF-directed stem cell lineage specification: dynamic composition regulates specific stages of skeletal myogenesis. Cell. Mol. Life Sci. 73, 3887-3896 (2016)

5. Wu, Z. et al. p38 and extracellular signal-regulated kinases regulate the myogenic program at multiple steps. Mol. Cell. Biol. 20, 3951-3964 (2000).

6. Pownall, M. E., Gustafsson, M. K. \& Emerson, C. P. Jr. Myogenic regulatory factors and the specification of muscle progenitors in vertebrate embryos. Annu. Rev. Cell Dev. Biol. 18, 747-783 (2002).

7. Chan, C. Y. et al. Identification of differentially regulated secretome components during skeletal myogenesis. Mol. Cell. Proteomics 10, M110 004804 (2011).

8. Garcia-Prat, L., Sousa-Victor, P. \& Munoz-Canoves, P. Proteostatic and metabolic control of stemness. Cell Stem Cell 20, 593-608 (2017).

9. Mauro, A. Satellite cell of skeletal muscle fibers. J. Biophys. Biochem. Cytol. 9 493-495 (1961).

10. Sincennes, M. C., Brun, C. E. \& Rudnicki, M. A. Concise review: Epigenetic regulation of myogenesis in health and disease. Stem Cells Transl. Med. 5, 282-290 (2016).

11. Black, B. L. \& Olson, E. N. Transcriptional control of muscle development by myocyte enhancer factor-2 (MEF2) proteins. Annu. Rev. Cell Dev. Biol. 14, 167-196 (1998)

12. Imbriano, C. \& Molinari, S. Alternative splicing of transcription factors genes in muscle physiology and pathology. Genes (Basel) 9, https://doi.org/10.3390/ genes9020107 (2018).

13. Ferri, P. et al. Expression and subcellular localization of myogenic regulatory factors during the differentiation of skeletal muscle C2C12 myoblasts. J. Cell. Biochem. 108, 1302-1317 (2009).

14. Lu, J., McKinsey, T. A., Zhang, C. L. \& Olson, E. N. Regulation of skeletal myogenesis by association of the MEF2 transcription factor with class II histone deacetylases. Mol. Cell 6, 233-244 (2000).

15. Edmondson, D. G., Lyons, G. E., Martin, J. F. \& Olson, E. N. Mef2 gene expression marks the cardiac and skeletal muscle lineages during mouse embryogenesis. Development 120, 1251-1263 (1994).

16. Ornatsky, O. I., Andreucci, J. J. \& McDermott, J. C. A dominant-negative form of transcription factor MEF2 inhibits myogenesis. J. Biol. Chem. 272, 33271-33278 (1997).

17. Johnson, J. E., Wold, B. J. \& Hauschka, S. D. Muscle creatine kinase sequence elements regulating skeletal and cardiac muscle expression in transgenic mice. Mol. Cell. Biol. 9, 3393-3399 (1989).

18. McCord, R. P., Zhou, V. W., Yuh, T. \& Bulyk, M. L. Distant cis-regulatory elements in human skeletal muscle differentiation. Genomics 98, 401-411 (2011).

19. Fujisawa-Sehara, A. et al. Differential trans-activation of muscle-specific regulatory elements including the mysosin light chain box by chicken MyoD, myogenin, and MRF4. J. Biol. Chem. 267, 10031-10038 (1992).

20. Estrella, N. L. et al. MEF2 transcription factors regulate distinct gene programs in mammalian skeletal muscle differentiation. J. Biol. Chem. 290, 1256-1268 (2015).

21. Grifone, R. et al. Six 1 and Six4 homeoproteins are required for Pax3 and Mrf expression during myogenesis in the mouse embryo. Development 132, 2235-2249 (2005)

22. Laclef, C. et al. Altered myogenesis in Six1-deficient mice. Development $\mathbf{1 3 0}$ 2239-2252 (2003)

23. Liu, Y., Chu, A., Chakroun, I., Islam, U. \& Blais, A. Cooperation between myogenic regulatory factors and SIX family transcription factors is important for myoblast differentiation. Nucleic Acids Res. 38, 6857-6871 (2010). 
24. Tobin, S. W. et al. Regulation of Hspb7 by MEF2 and AP-1: implications for Hspb7 in muscle atrophy. J. Cell. Sci. 129, 4076-4090 (2016).

25. Alli, N. S. et al. Signal-dependent fra-2 regulation in skeletal muscle reserve and satellite cells. Cell Death Dis. 4, e692 (2013).

26. Suzuki, A., Pelikan, R. C. \& Iwata, J. WNT/beta-catenin signaling regulates multiple steps of myogenesis by regulating step-specific targets. Mol. Cell. Biol. 35, 1763-1776 (2015).

27. Suzuki, A., Scruggs, A. \& Iwata, J. The temporal specific role of WNT/betacatenin signaling during myogenesis. J. Nat. Sci. 1, e143 (2015).

28. Otto, A. et al. Canonical Wnt signalling induces satellite-cell proliferation during adult skeletal muscle regeneration. J. Cell. Sci. 121, 2939-2950 (2008).

29. Kollias, H. D., Perry, R. L., Miyake, T., Aziz, A. \& McDermott, J. C. Smad7 promotes and enhances skeletal muscle differentiation. Mol. Cell. Biol. 26, 6248-6260 (2006).

30. Cohen, T. V., Kollias, H. D., Liu, N., Ward, C. W. \& Wagner, K. R. Genetic disruption of Smad7 impairs skeletal muscle growth and regeneration. J. Physiol. 593, 2479-2497 (2015).

31. Miyake, T., Alli, N. S. \& McDermott, J. C. Nuclear function of Smad7 promotes myogenesis. Mol. Cell. Biol. 30, 722-735 (2010).

32. Edlund, S. et al. Interaction between Smad7 and beta-catenin: importance for transforming growth factor beta-induced apoptosis. Mol. Cell. Biol. 25 1475-1488 (2005)

33. Ehyai, S. et al. A p38 mitogen-activated protein kinase-regulated myocyte enhancer factor 2-beta-catenin interaction enhances canonical Wnt signaling. Mol. Cell. Biol. 36, 330-346 (2016).

34. Ehyai, S. et al. FMRP recruitment of beta-catenin to the translation pre-initiation complex represses translation. EMBO Rep. 19, https://doi.org/10.15252/ embr.201745536 (2018).

35. Valenta, T., Hausmann, G. \& Basler, K. The many faces and functions of betacatenin. EMBO J. 31, 2714-2736 (2012).

36. Chamberlain, J. S., Jaynes, J. B. \& Hauschka, S. D. Regulation of creatine kinase induction in differentiating mouse myoblasts. Mol. Cell. Biol. 5, 484-492 (1985).

37. Sartorelli, V. \& Puri, P. L. Shaping gene expression by landscaping chromatin architecture: lessons from a master. Mol. Cell 71, 375-388 (2018).

38. Kim, C. H., Neiswender, H., Baik, E. J., Xiong, W. C. \& Mei, L. Beta-catenin interacts with MyoD and regulates its transcription activity. Mol. Cell. Biol. 28, 2941-2951 (2008).

39. Kim, S., Xu, X., Hecht, A. \& Boyer, T. G. Mediator is a transducer of Wnt/betacatenin signaling. J. Biol. Chem. 281, 14066-14075 (2006).

40. Furumoto, T. et al. A kinase subunit of the human mediator complex, CDK8, positively regulates transcriptional activation. Genes Cells 12, 119-132 (2007).

41. Tsivitse, S. Notch and Wnt signaling, physiological stimuli and postnatal myogenesis. Int. J. Biol. Sci. 6, 268-281 (2010).

42. Cisternas, P., Henriquez, J. P., Brandan, E. \& Inestrosa, N. C. Wht signaling in skeletal muscle dynamics: myogenesis, neuromuscular synapse and fibrosis. Mol. Neurobiol. 49, 574-589 (2014).
43. Snyder, C. M. et al. MEF2A regulates the Gt12-Dio3 microRNA mega-cluster to modulate WNT signaling in skeletal muscle regeneration. Development 140 31-42 (2013).

44. Vallee, A., Lecarpentier, Y., Guillevin, R. \& Vallee, J. N. Interactions between TGFbeta1, canonical WNT/beta-catenin pathway and PPAR gamma in radiationinduced fibrosis. Oncotarget 8, 90579-90604 (2017).

45. Kelleher, R. J. 3rd, Flanagan, P. M. \& Kornberg, R. D. A novel mediator between activator proteins and the RNA polymerase II transcription apparatus. Cell 61, 1209-1215 (1990).

46. Kornberg, R. D. Mediator and the mechanism of transcriptional activation. Trends Biochem. Sci. 30, 235-239 (2005).

47. Malik, S. \& Roeder, R. G. Dynamic regulation of pol II transcription by the mammalian Mediator complex. Trends Biochem. Sci. 30, 256-263 (2005).

48. Kim, Y. J., Bjorklund, S., Li, Y., Sayre, M. H. \& Kornberg, R. D. A multiprotein mediator of transcriptional activation and its interaction with the C-terminal repeat domain of RNA polymerase II. Cell 77, 599-608 (1994).

49. Baek, H. J., Kang, Y. K. \& Roeder, R. G. Human Mediator enhances basal transcription by facilitating recruitment of transcription factor $\| B$ during preinitiation complex assembly. J. Biol. Chem. 281, 15172-15181 (2006).

50. Mittler, G., Kremmer, E., Timmers, H. T. \& Meisterernst, M. Novel critical role of a human Mediator complex for basal RNA polymerase II transcription. EMBO Rep. 2, 808-813 (2001).

51. Taatjes, D. J. The human Mediator complex: a versatile, genome-wide regulator of transcription. Trends Biochem. Sci. 35, 315-322 (2010).

52. Soutourina, J., Wydau, S., Ambroise, Y., Boschiero, C. \& Werner, M. Direct interaction of RNA polymerase $\|$ and mediator required for transcription in vivo. Science. 331, 1451-1454 (2011).

53. Thompson, C. M. \& Young, R. A. General requirement for RNA polymerase ॥ holoenzymes in vivo. Proc. Natl Acad. Sci. USA 92, 4587-4590 (1995).

54. Myers, L. C. \& Kornberg, R. D. Mediator of transcriptional regulation. Annu. Rev. Biochem. 69, 729-749 (2000).

55. Bjorklund, S. \& Gustafsson, C. M. The yeast Mediator complex and its regulation. Trends Biochem. Sci. 30, 240-244 (2005).

56. Soutourina, J. Transcription regulation by the Mediator complex. Nat. Rev. Mol. Cell Biol. 19, 262-274 (2018).

57. Holstege, F. C. et al. Dissecting the regulatory circuitry of a eukaryotic genome. Cell. 95, 717-728 (1998).

58. Paulino, A. C. \& Okcu, M. F. Rhabdomyosarcoma. Curr. Probl. Cancer. 32, 7-34 (2008).

59. Dionyssiou, M. G., Ehyai, S., Avrutin, E., Connor, M. K. \& McDermott, J. C. Glycogen synthase kinase 3beta represses MYOGENIN function in alveolar rhabdomyosarcoma. Cell Death Dis. 5, e1094 (2014).

60. Rampalli, S. et al. p38 MAPK signaling regulates recruitment of Ash2Lcontaining methyltransferase complexes to specific genes during differentiation. Nat. Struct. Mol. Biol. 14, 1150-1156 (2007). 